\section{$\underset{\substack{\text { hommes } \\ \text { \& migrations }}}{ }$}

\section{Hommes \& migrations}

Revue française de référence sur les dynamiques

migratoires

1307 | 2014

L'Afrique qualifiée dans la mondialisation

\title{
Le retour difficile des jeunes Maliens formés en l'Amérique du Nord
}

\section{Kévin Mary}

\section{OpenEdition \\ Journals}

\section{Édition électronique}

URL : http://journals.openedition.org/hommesmigrations/2876

DOI : 10.4000/hommesmigrations.2876

ISSN : 2262-3353

\section{Éditeur}

Musée national de l'histoire de l'immigration

Édition imprimée

Date de publication : 1 juillet 2014

Pagination : $39-46$

ISBN : 978-2-919040-28-5

ISSN : $1142-852 X$

\section{Référence électronique}

Kévin Mary, "Le retour difficile des jeunes Maliens formés en l'Amérique du Nord », Hommes \& migrations [En ligne], 1307 | 2014, mis en ligne le 01 juillet 2017, consulté le 19 avril 2019. URL : http:// journals.openedition.org/hommesmigrations/2876; DOI : 10.4000/hommesmigrations.2876 


\title{
LE RETOUR DIFFICILE DES JEUNES MALIENS FORMÉS EN AMÉRIQUE DU NORD
}

par KEVIN MARY, doctorant en géographie, UMR ESO et MIGRINTER.

\author{
Au Mali, les familles appartenant à l'élite ont coutume \\ d'envoyer leurs enfants étudier à l'étranger, afin qu'ils \\ acquièrent une qualité de formation qui leur semble faire défaut \\ dans leur pays. Mais ces jeunes diplômés peinent à conserver \\ le statut social de leurs parents. La désertion de l'enseignement \\ supérieur au Mali cristallise les impasses de la société \\ malienne. Car le retour s'avère difficile. Les carrières locales \\ sont mal rémunérées. Et l'entregent, bien plus que les diplômes, \\ demeure la clé des cercles du pouvoir.
}

Le retour au Mali des jeunes formés en Amérique du Nord fait question. Issus des "grandes familles" de la bourgeoisie bamakoise, ces étudiants, dont les parents occupent des positions de premier plan dans l'appareil d'État (ministres, hauts fonctionnaires, hauts gradés de l'armée, directeurs nationaux, etc.), ont étudié dans les campus universitaires nord-américains, avant de revenir s'installer à Bamako. En portant la réflexion sur les pratiques de retour de ces diplômés, il s'agit de s'intéresser à un enjeu relativement peu abordé par les travaux sur les migrations étudiantes, plus souvent analysées sous l'angle de l'installation - durable - dans le pays d'études ${ }^{1}$. De plus, l'insertion de ces jeunes issus de "grandes familles" sur le marché du travail de leur pays d'origine se pose en des termes particuliers, renvoyant moins à la mobilité sociale dans la mesure où l'acquisition de leurs diplômes pourrait au mieux leur permettre de conserver le même statut social que leurs parents - quà la reproduction des groupes sociaux dominants dans les structures du pouvoir politique et économique malien. Le terme d'“hégémonie" se réfère ici aux travaux d'Antonio Gramsci. Il permet de rappeler que les stratégies déployées par ces familles n'arrivent pas toujours à porter leurs fruits. D'autres acteurs leur disputent en effet les places liées aux postes de pouvoir. Leur domination n'est en ce sens qu'un "projet social en voie de réalisation (...), qui s'oppose à des projets hégémoniques rivaux²". 
Fondé sur des enquêtes de terrain de plusieurs mois à Bamako, au cours de séjours répétés ${ }^{3}$, cet article souhaite mettre en tension les stratégies spatiales déployées par les membres de l'élite politico-économique pour envoyer leurs enfants étudier à l'étranger, au regard des difficultés que ces derniers rencontrent sur le marché du travail malien, une fois leurs diplômes en poche.

\section{Genèse et développement de la "question scolaire" au Mali}

À l'indépendance du pays en 1960, il n'existait aucune structure d'enseignement supérieur. Sur la base de l'option socialiste dans laquelle s'est engagé le pays, la réforme de 1962 visait à l'autonomie en matière de ressources humaines nationales, dans le cadre d'une économie planifiée dont l'État était le principal agent, et faisait le choix de former sur place ses cadres supérieurs par le biais de grandes écoles. Pendant une vingtaine d'années, l'État malien a systématiquement intégré les diplômés de ses écoles, mais également ceux formés à l'étranger, compte tenu du manque de diplômés dans le pays durant cette période au regard des besoins en personnels. L'anthropologue malien Issiaka Bagayogo rapporte que la majorité des cadres formés à l'étranger provenaient des pays de l'Est, en particulier de l'ex-URSS, tandis qu'une minorité avait été formée en Occident ${ }^{4}$.

Les années 1980 marquent le passage du Mali sous ajustement structurel, qui remet en cause l'intégration systématique des diplômés dans la fonction publique. L'État ne recrute plus désormais que sur concours, et selon ses besoins. Parallèlement, les effectifs d'étudiants croissent de manière rapide et régulière, dépassant largement les capacités d'accueil des grandes écoles. L'image de l'ensei- gnement supérieur malien comme lieu principal de formation de l'élite nationale est remis en cause et la "question scolaire" arrive sur le devant de la scène politique malienne. Cette étape marque le début de la désorganisation du système d'enseignement supérieur. Elle s'observe d'abord à l'échelle nationale, par l'inconsistance des projets politiques en matière d'éducation autres que ceux qui se fondent sur la seule logique économique, dans le cadre des plans d'ajustements structurels de réduction des dépenses publiques. Cette politique a conduit à une succession de "crises scolaires", dont l'une d'elles servira de moteur à la chute du régime de Moussa Traoré en 1991. À l'échelle locale, le désordre s'affiche avant tout dans les calendriers universitaires. Les mouvements de grèves conduits régulièrement par les étudiants ou les enseignants, voire les fermetures administratives décrétées par le gouvernement, mettent à rude épreuve l'organisation des années scolaires.

C'est dans ce contexte de crise qu'est née en 1996 l'université du Mali5. Face à l'augmentation toujours importante du nombre de bacheliers, elle se veut un complément des grandes écoles. En 2011, le nombre d'étudiants inscrits dans l'enseignement supérieur continue d'exploser et passe à environ $80000^{6}$. La "question scolaire" ${ }^{\text {" parait }}$ encore aujourd'hui loin d'être résolue. Les années scolaires partielles se banalisent, tandis que les "années blanches" n’ont pas disparu.

\section{L'enseignement supérieur malien face à la démission des élites}

Plus de cinquante ans après sa création, la dégradation du système d'enseignement supérieur malien aura finalement consacré la faiblesse des structures nationales de la formation des élites ${ }^{8}$. Les familles

3. Au total, les enquêtes de terrain ont duré plus d'un an et reposent sur une centaine d'entretiens semi-directifs, ainsi que sur des observations, suivant une démarche davantage ethnographique. 4. Issiaka Bagayogo, "La crise de l'enseignement supérieur au Mali", in Adebayo Olukoshi, Mohamed Chérif Diarra (dir.), Enjeux du financement et de la planification de l'éducation en Afrique, Dakar, ADEA, 2007. 5. Si la loi n93-06o portant la création de l'université du Mali a été votée dès 1993, ce n'est cependant qu'en 1996 et avec le décret n96-156/P-RM du 23 mai, fixant l'organisation et les modalités de fonctionnement de l'université du Mali, qu'elle a ouvert ses portes. 6. Source : rectorat de l'université de Bamako. 7. Soit les réponses à apporter face à l'ampleur de la crise scolaire. 8. Des établissements privés d'enseignement supérieur se sont bien développés, mais ils demeurent confrontés au problème de l'homologation de leurs diplômes, susceptible de remettre en cause la participation de leurs étudiants aux concours et aux examens d'État. 
qui en ont les moyens mettent désormais en place des stratégies d'exode scolaire, afin d'envoyer leurs enfants étudier à létranger. L'enseignement supérieur malien se trouve ainsi déconsidéré par les groupes sociaux dominants, qui reconnaissent dans les pays étrangers de nouveaux pôles d'excellence en matière de formation. Curieux destin que celui des dirigeants maliens qui, en charge notamment de la question scolaire depuis plusieurs décennies, choisissent de ne pas y investir à titre personnel, lorsque l'enjeu est celui de l'avenir de leur progéniture. "Si les étudiants ne veulent pas étudier, laissez-les continuer à grever, nous nos enfants étudient à l'extérieur, ils reviendront vous gouverner comme nous nous l'avons fait ${ }^{9}$." Ces propos tenus en 1994 par Ibrahim Boubacar Keita - Premier ministre à lépoque - à la télévision nationale, alors qu'il faisait face à un mouvement de grève des étudiants, suggèrent assez bien l'état d'esprit qui anime les dirigeants du pays quant à la question de léducation en général, et de celle de leurs enfants en particulier. Elle en dit long également sur leur conception du pouvoir, qui dans cet extrait se mêle étroitement à celui du savoir. De tels propos dans la bouche de l'actuel président de la République invitent donc à s'interroger - à l'heure où le Mali revêt de nouveau les atours de la "démocratie" - sur le rapport au pouvoir du nouveau chef de l'État et sur la relation qu'il entretient avec le système d'enseignement supérieur malien.

\section{L'internationalisation des études supérieures}

Bien que le Mali indépendant - sous l'impulsion du régime socialiste de Modibo Keïta - ait opté pour une politique de formation locale de ses cadres, via la réforme de l'éducation de 1962, il faut accorder au phénomène des études à l'étranger un principe d'antériorité. En effet, avant l'indépendance du pays en 1960, l'État colonial s'était déjà mué en État formateur : les premiers étudiants maliens ont étudié soit en France, soit au Sénégal, à l'École normale William-Ponty, lorsque le colon avait besoin d'intermédiaires indigènes dans son administration.

L'option socialiste envisagée au moment de l'indépendance, et en pleine guerre froide, a permis de développer les liens avec les pays du bloc de l'Est, qui deviendront à ce moment-là le premier pôle de formation des Maliens. Pour autant, la France continue de former des étudiants venus du Mali, en nombre certes moins important que dans les pays de l'Est, comme l'a rappelé Issiaka Bagayogo, mais de manière continue ${ }^{10}$. L'arrivée au pouvoir des militaires en 1968 semble marquer une diversification des destinations pour études, sans pour autant remettre profondément en cause le schéma préexistant.

Sous des auspices désormais plus "libéraux", les ÉtatsUnis et le Canada s'imposent progressivement comme des destinations de premier plan pour les études. Deux raisons
L'option socialiste envisagée au moment de l'indépendance, et en pleine guerre froide, a permis de développer les liens avec les pays du Bloc de l'Est, qui deviendront à ce moment-là le premier pôle de formation des Maliens. principales peuvent être évoquées ici. La première renvoie à l'insertion du Mali dans une économie de marché, où ces deux pays cherchent à attirer de nouveaux étudiants, comme le souligne le cinéaste et homme politique Cheick Oumar Sissoko: "Je crois que ces pays ont compris qu'il était intéressant de s'ouvrir aux pays africains qui ne font pas partie de leur marché potentiel." Le romancier Ousmane Diarra note qu'il y a eu selon lui un "mouvement chez les familles nanties, qui se sont tournées vers l'Amérique à partir de 198311”, année qui marque l'intervention de la Banque mondiale et du FMI au Mali, dans le cadre des programmes d'ajustements structurels. La seconde raison - et peutêtre la plus importante - tient à la mise en place en France de politiques de contrôle des flux migratoires à partir de 1974, avec l'arrêt de l'immigration de travail. Pour les étudiants notamment, ces politiques restrictives, à la suite desquelles le continent européen est en passe de devenir une 
"forteresse", se traduisent concrètement par toute une série de tracasseries administratives apparentant les démarches d'obtention d'un visa d'étudiant à un véritable "parcours du combattant ${ }^{12 "}$. Ces nouvelles conditions

Les liens historiques unissant

le Mali à son ancienne puissance coloniale resteraient

forts et ne remettraient

que partiellement en cause

l'attrait qu'elle suscite

auprès des groupes sociaux

dominants.

ne remettent cependant pas fondamentalement en cause l'attrait pour cette destination historique : la France reste l'une des destinations d'études privilégiées auprès des membres des classes dominantes.
La chute du régime militaire en 1991 préfigure une période propice aux changements. L'avènement de la "démocratie" n'a pourtant pas d'influence considérable sur les destinations de l'exode scolaire, du moins en ce qui concerne les élites nationales ${ }^{13}$. Un nombre croissant d'étudiants maliens se rend aux États-Unis et au Canada à partir de cette période ${ }^{14}$. La France, malgré sa politique de fermeture, demeure, selon les estimations disponibles, la première destination d'études des Maliens dans le monde ${ }^{15}$. Les liens historiques unissant le Mali à son ancienne puissance coloniale resteraient forts et ne remettraient que partiellement en cause l'attrait qu'elle suscite auprès des groupes sociaux dominants. C'est du moins ce qu'affirme l'avocat d'affaires et homme politique Abdoulaye Garba Tapo ${ }^{16}$ : “Aujourd'hui l'idéal, pour ceux qu'on peut considérer comme les plus nantis, c'est d'envoyer leurs enfants à l'étranger. Ce qui les détourne de la France, c'est les conditions d'accès et d'obtention du visa. (...) Mais il faut reconnaître que les Américains ont fait plus d'ouverture, surtout ces dernières années, pour ceux qui veulent aller étudier. Il y a maintenant une tendance chez les Américains à donner systématiquement des visas de cinq ans. Or, en France, c'est quand même toujours très très strict et c'est ça qui au fond frustre un peu les gens. (...) Mais la France reste quand même la destination privilégiée, la cible idéale."

À l'issue de ce tour d'horizon, il apparaît que les principales destinations de l'émigration pour études au Mali sont faites de ruptures, mais aussi de continuité. Les évolutions politiques au Mali ont participé au redéploiement spatial de ces migrations, sans pour autant remettre en cause la tendance historique aux études en France ${ }^{17}$. La "quête d'internationalisation" des élites nationales en matière d'éducation n'est, par ailleurs, pas sans rappeler le rapport de force symbolique qui se joue entre les nations. En vertu du tropisme de l'attrait des pays développés, la France, les États-Unis et le Canada constituent ainsi un trio de tête.

\section{Des carrières locales déconsidérées}

"Tout ça pour des enfants dont on n'entend même plus parler après." Cette phrase entendue à Bamako suggère bien le rapport particulier entre les études estimées coûteuses des jeunes parfois péjorativement considérés comme des "enfants gâtés" ou des "fils à papa” et leur participation, en retour, à la vie économique et politique du pays. Sur un plan plus théorique, les difficultés que semblent rencontrer ces jeunes au moment de leur retour dans le pays d'origine posent la question de la reproduction des positions dominantes. Elles invitent également à s'interroger sur les déterminants de la production des inégalités et des rapports de domination propres à la société malienne.

En somme, il ne s'agit pas ici de développer une approche "hors sol", oublieuse d'un contexte natio- 
nal où les populations demeurent dans une large part soumises à la précarité matérielle, voire à "l'insécurité alimentaire"18". En comparaison, ces jeunes paraissent relativement épargnés.

Lorsque l'un de nos informateurs évoque le retour au Mali de ses cousins et de ses amis proches partis étudier aux États-Unis ou au Canada, il les décrit comme étant "aigris". Il pointe ici les bénéfices estimés "maigres" des études à létranger pour des jeunes ayant toujours été habitués à un mode de vie aisé, en indiquant que "même s'ils sont cadres, combien est-ce qu'ils gagnent? Même si ce sont des hauts cadres, ils vont gagner au maximum 700000 ou 800000 francs CFA, ce qui représente environ 1000 euros", ajoutant que ce n'est "pas au Mali qu'on gagne le plus d'argent $t^{19}$.

Nous avons retenu ici deux cas de figures qui permettent d'illustrer ce propos, à travers le parcours de deux jeunes de bonne famille, de retour à Bamako après des études supérieures aux ÉtatsUnis.

Ibrahim $^{20}$, aujourd'hui âgé de 43 ans, est rentré d'Amérique depuis plusieurs années avec un bachelor de gestion en poche, délivré par le Northeastern Oklahoma A\&M College et entièrement financé par ses parents. Il travaille aujourd'hui pour l'ambassade des États-Unis à Bamako, où il occupe un poste de magasinier responsable des approvisionnements en denrées périssables de l'ambassade ${ }^{21}$. L'une de ses cousines considère qu'il "a vraiment un rôle de commis là-bas, pas de cadre en tout cas ${ }^{22 "}$. Embauché en qualité d'employé local, son contrat de travail s'avère peu avantageux, à la fois en termes de rémunération et de conditions de travail ${ }^{23}$. Pourtant, Ibrahim est issu d'une "grande famille". Son père a été l'un des principaux militants de l'Union soudanaise-Rassemblement démocratique africain (US-RDA), le parti au pouvoir au moment de l'indépendance. Il a occupé des postes de diplomate

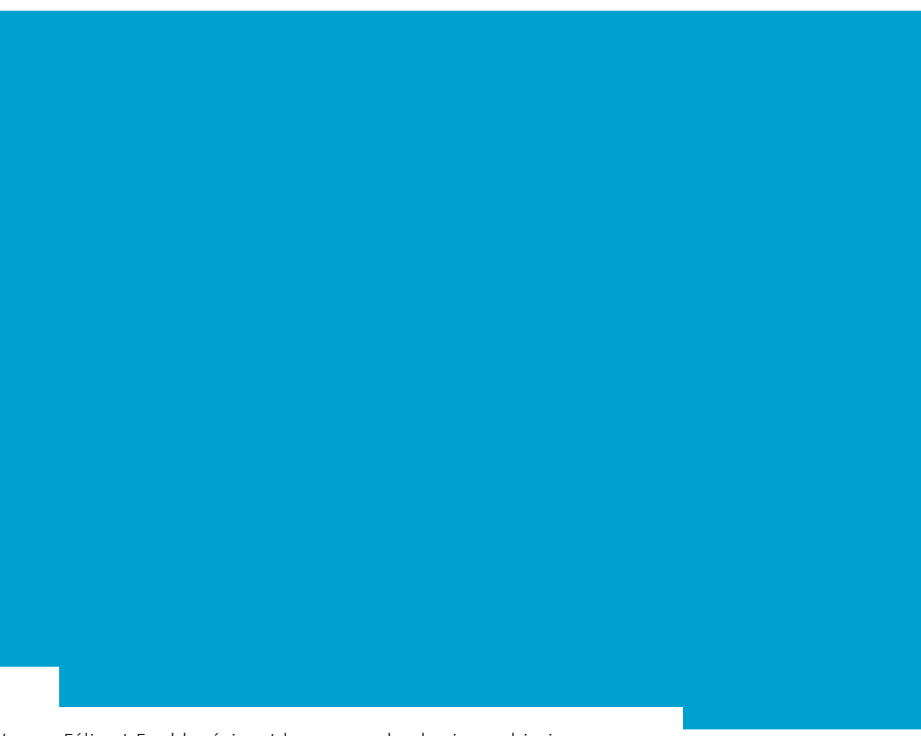

Félix et Freddy révisent leur cours de physique-chimie. Ils sont en licence 2 à l'université Félix-Houphouët-Boigny. Abidjan, mai 2014 @ Camille Millerand

sous le régime de Modibo Keïta, puis sous celui de Moussa Traoré. Ibrahim est l'un de ses fils né d'un second mariage. Sa mère était, par ailleurs, une femme influente sous le régime de Moussa Traoré, lorsqu'elle était l'une des dirigeantes de l'Union nationale des femmes du Mali, l'un des organes du parti unique de l'Union démocratique du peuple malien (UDPM).

\section{Des emplois mal rémunérés}

Arrivée au Mali en 2010 avec un MBA en finance de l'université de Houston (Texas), Habibatou, âgée de 25 ans, a connu un parcours similaire. Après avoir cherché sans succès du travail dans le secteur des mines, puis à l'Union économique et monétaire ouest-africaine (UEMOA), elle a finalement réussi à obtenir un stage dans une banque, grâce notamment aux relations de ses parents ${ }^{24}$. À l'issue de ce stage rémunéré l'équivalent d'un peu moins de 80 euros par mois, la banque l'embauche en tant que responsable des audits internes dans l'une de ses agences bamakoises pour un salaire 
mensuel de 330000 francs CFA (environ 500 euros), qualifié de "léger" par son père. Ce dernier avait sans doute espéré mieux pour sa fille, au regard de son parcours personnel. Il a été directeur national de l'urbanisme et de l'habitat de 1979 à 1988, un poste clé dans l'administration, puisqu'il se situe hiérarchiquement juste en dessous de celui du ministre et de son cabinet ${ }^{25}$. Au cours de son activité, son père met notamment en place le premier projet urbain du Mali en 1980, placé sous l'égide de la Banque mondiale. La mère d'Habibatou était en 2009 directrice de cabinet du ministre de la Justice. Les deux parents confient "mettre tous [leurs] efforts" dans l'éducation de leurs enfants. Ils n'hésitent pas à envoyer par mandats d'importantes sommes d'argent à leurs filles en Amérique, au risque parfois de mettre en difficulté leurs propres affaires au Mali ${ }^{26}$. Les parcours de ces deux jeunes résonnent ainsi de manière paradoxale, au regard

Le simplefait d'avoir accumulé leurs parents, mais égalesuffisamment d'argent pour ment du fort investisseenvoyer ses enfants étudier dans

les prestigieuses universités occidentales, et l'acquisition du capital scolaire qui en découle, ne suffisent visiblement pas à pérenniser le processus engendré par les parents. au chercheur de cerner rapidement les contours de ces groupes sociaux. Peuplé d'un peu plus de 15 millions d'habitants en 2011, le Mali est un État de petite taille. Avec une population de surcroît études ont fait l'objet. Les deux situations décrites ne nous semblent pas liées à un biais méthodologique, dans la mesure où la taille restreinte des classes domi-
Sortie de cours sur le campus universitaire Félix-HouphouëtBoigny (Abidjab), mai 2014 @ CAmille Millerand non seulement de ceux de

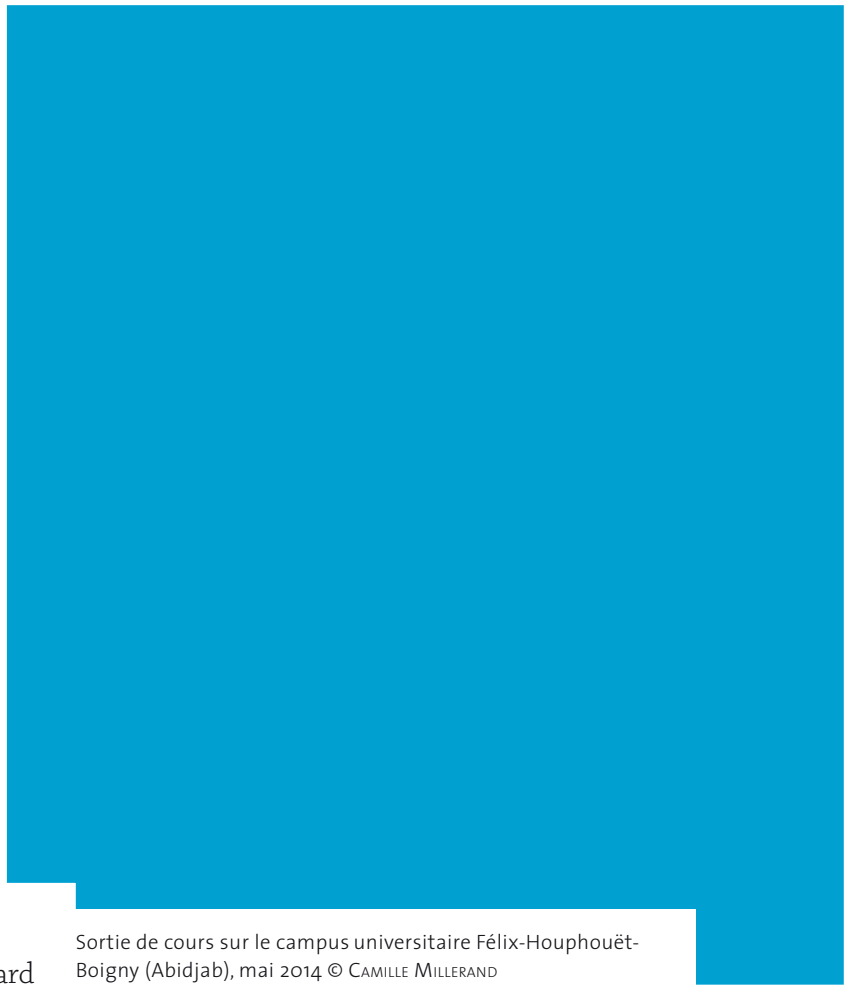

particulièrement jeune et peu éduquée ${ }^{27}$, la classe dominante malienne - concentrée dans la capitale - prend un caractère volontiers "intimiste" 28. ent expliquer l'échec relatif de ces stratégies éducatives ? Quelles médiations empêchent ici la reproduction sociale? Quels sont, in fine, les processus qui permettent l'accumulation ? Le simple fait d'avoir accumulé suffisamment d'argent pour envoyer ses enfants étudier dans les prestigieuses

25. Les postes de directeurs nationaux des différents ministères sont l'objet d'âpres batailles politiques pour leur contrôle. II s'agit du poste le plus élevé dans l'administration d'État. Cette position stratégique dans l'appareil d'État s'illustre notamment par son appellation officieuse de “bureau de placement"... 26. Alors que la structure de formation professionnelle créée par le père d'Habibatou à Bamako, dont il est le directeur général, rencontrait des difficultés financières importantes, rendant notamment impossible le paiement des salaires de ses employés, l'assistant en formation de la structure se souvient d'avoir été commissionné à plusieurs reprises par M. Diarra pour envoyer des virements bancaires d'un million de francs CFA (environ 1500 euros) vers les États-Unis. 27. Selon les chiffres fournis par la Banque mondiale, la population des moins de 15 ans s'élevait à $47 \%$ en 2010, et le taux d'alphabétisation à $31 \%$. 28. Voir à ce sujet Jean-François Bayart, L'État en Afrique. La politique du ventre, Paris, Fayard, 2006, p. 201. Cet article s'inscrit dans le cadre d'un travail de thèse portant sur les migrations pour études en Amérique du Nord dans les familles des élites maliennes. Nous avons ainsi pu conduire une centaine d'entretiens et constater ce fort degré d'interconnaissance. 
universités occidentales, et l'acquisition du capital scolaire qui en découle, ne suffisent visiblement pas à pérenniser le processus engendré par les parents. D'un autre côté, les diplômes acquis en Amérique ont néanmoins une certaine valeur "marchande", puisqu'ils permettent d'obtenir un travail, même s'il s'agit au final d'un poste subalterne. Ainsi, on comprend mieux pourquoi une partie de ces jeunes décident de rester dans le pays qui les a formés à l'issue de l'obtention de leur diplôme ${ }^{29}$. En fait, la réussite du retour de ces jeunes au pays dépend grandement de la capacité de leurs parents à les insérer dans les réseaux clientélistes qui structurent la société malienne. Dans le cadre d'un rapport au pouvoir fortement personnalisé aux racines précoloniales ${ }^{30}$, l'efficacité d'une recommandation pour obtenir un poste est fonction des cercles du pouvoir auxquels on s'adresse. Comme l'indique Issiaka Bagayogo: "L'exercice de la domination (...) s'effectue à partir de l'épicentre que constitue le chef de l'État, autour duquel se forme un noyau central. (...) De là, et par cercles concentriques, le pouvoir


cette logique, il n'est pas surprenant de constater l'empressement de cette mère de famille cadre au Trésor public, pour que son fils rentre du Canada avec son diplôme pendant qu'elle et son mari sont "encore aux affaires" et qu'ils peuvent "faire quelque chose pour lui i2", ou encore le succès rencontré par les filles de l'ancien Président Amadou Toumani Touré qui, après des études en communication au Canada, ont monté l'agence Océan Communication, et dont "on sait que tous les marchés de l'État passent par eux", comme le confiait un journaliste malien, bon connaisseur des milieux politiques bamakois. C'est bien l'importance du rapport à l'État qui se dessine en filigrane. Il apparaît que ce sont les relations entretenues par les acteurs avec l'appareil d'État qui confèrent la capacité de s'enrichir au Mali ${ }^{33}$, comme ailleurs en Afrique $^{34}$. Ces relations s'avèrent à la fois fragiles et difficilement pérennes, car soumises aux aléas conjoncturels des changements de poste et de gouvernement. Aussi, les relations de pouvoirs ne tiennent-elles pas à grandchose : la perte d'un poste, ou encore un décès sont susceptibles de faire perdre l'entregent et de remettre en cause le statut social. Ne diton pas au Mali d'un nouveau
Même au sommet de l'État, la reproduction ne parait pas garantie. Le risque d'une dislocation des relations sociales semble bien présent, et avec elle, la capacité d'intégrer les réseaux qui opèrent sur des bases clientélistes se voit amputée. pouvoir qu'il "vient avec ses gens" ? À cet égard, le journaliste interrogé parle de situation de "rente", en décrivant l'inquiétude d'Amadou Toumani Touré et de sa femme pour l'avenir de leurs filles à l'issue des élections de 2012, qui n'ont finalement pas eu lieu, suite à un coup d'État ${ }^{35}$ : "Ils sont persuadés que si eux ne parviennent pas à mettre le maximum de sécurité financière autour de leurs enfants, personne d'autre ne le fera à leur place." Même au sommet de l'État, la reproduction ne parait pas garantie. Le risque d'une dislocation des relations sociales semble bien présent, et avec elle, la capacité d'intégrer les réseaux qui opèrent sur des bases clientélistes se voit amputée.

\section{Conclusion}

L'analyse du développement de la question scolaire au Mali fait porter une partie de la responsabilité aux élites au pouvoir, qui n'ont pas hésité, lorsque les conditions d'un enseignement supérieur local

29. Bien que l'on ne connaisse pas le nombre des retours de diplômés maliens d'Amérique. Cette statistique se heurte à un problème à la fois de définition (comment rendre compte par exemple des phénomènes de circulation ?) et de mesure, car le retour ne s'accompagne pas forcément d'un acte administratif. Néanmoins, nos informateurs laissent entendre qu'environ la moitié des diplômés maliens d’Amérique ne rentrent pas à l'issue de leur "graduation”. 30. Shaka Bagayogo, "L'État au Mali. Représentation, autonomie et mode de fonctionnement", in Emmanuel Terray (dir.), L'État contemporain en Afrique, Paris, L’Harmattan, 1987 ; Claude Fay, "La démocratie au Mali, ou le pouvoir en pâture”, in Cahiers d'études africaines, vol. 35, n 137, 1995. 31. Shaka Bagayogo, "L'État au Mali. Représentation, autonomie et mode de fonctionnement”, art. cité, p. 110. 32. Source : entretien réalisé à Bamako le 17 juillet 2011. 33. Jean-Loup Amselle, “La corruption et le clientélisme au Mali et en Europe de l'Est : quelques points de comparaison”, in Cahiers d'études africaines, vol. 32, n 128, 1992 ; Shaka Bagayogo, “'ĹEtat au Mali. Représentation, autonomie et mode de fonctionnement", art. cité. ; Claude Fay, “La démocratie au Mali, ou le pouvoir en pâture”, art. cité 34. Jean-François Bayart, L'État en Afrique. La politique du ventre, op. cit., p. 119. 35. Le 22 mars 2012, et un mois seulement avant l'échéance de l'élection présidentielle, un coup d'État militaire a destitué Amadou Toumani Touré. 
de qualité se délitaient, à investir dans le label étranger - qu'il soit français ou américain - pour leurs propres enfants. Les propos pour le moins ambivalents tenus par l'actuel président de la République au sujet du problème crucial de l'école suggèrent que la résolution de la crise scolaire risque fort d'être laissée aux promesses électorales. Les "enfants des riches" n'ont pour autant pas encore gagné. Lorsqu'ils retournent aujourd'hui dans leur pays d'origine, leurs diplômes acquis à l'étranger s'avèrent loin de leur garantir la position sociale acquise par leurs parents, dès lors que ces derniers ne peuvent plus assurer à leur progéniture l'accès aux réseaux de l'appareil d'État. Les équilibres paraissent fragiles dans un pays où le statut social se mesure à la capacité des acteurs à se rapprocher du pouvoir situé au cœur de l'État, et dont la personne du président de la République fait figure de noyau central. Mais l'État doit également être pensé comme la "condensation d'un rapport de forces" entre classes et fractions de classes, à la manière de Nicos Poulantzas ${ }^{36}$, où de "nouvelles figures de la réussite et du pouvoir ${ }^{37 "}$ sont susceptibles d'émerger. On a, en effet, assisté ces derniers temps au Mali à des changements importants, parmi lesquels le retour des militaires sur le devant de la scène à travers la figure du capitaine - aujourd'hui général Amadou Haya Sanogo, ce qui a pu être considéré un moment comme une revanche des cadets sociaux sur un ordre politique sclérosé38, ou encore l'arrivée de certains représentants de l'islam malien dans le champ politique, avec la création d'un ministère des Affaires religieuses et du Culte ${ }^{39}$.

Aussi sommes-nous enclin à penser la classe dominante malienne comme étant en voie de formation, comme le suggère Jean-François Bayart à propos du Cameroun ${ }^{40}$, plus qu'en tant que système achevé. Cet "inachèvement" n'exclut pas néanmoins des tentatives qui pourraient, à l'avenir, s'avérer payantes. Quelques trajectoires personnelles le prouvent. La candidature de Cheick Boucadry Traoré, fils de Moussa Traoré, à lélection présidentielle de 2013 en est un indice. L'ascension de Moussa Mara dans le gouvernement actuel, ce fils d'un ancien ministre de la Justice et proche de Moussa Traoré, en est un autre. Mais c'est bien la candidature de Karim Keïta, fils du président Ibrahim Boubacar Keïta et formé au Canada, aux élections législatives de 2013, qui suscite le plus de commentaires : son accession à un mandat électoral constitue une première dans le pays. Cependant, la stratification sociale malienne ne semble pas encore avoir atteint le degré de fermeture que l'on peut observer dans les sociétés dites "développées", comme le montrent par exemple les travaux de Michel et Monique Pinçon-Charlot ${ }^{41}$ sur la grande-bourgeoisie française. 